\title{
Novel tumor markers in the serum of testicular germ cell cancer patients: a review
}

This article was published in the following Dove Press journal:

Current Biomarker Findings

9 September 2014

Number of times this article has been viewed

\section{Isabella Syring \\ Stefan C Müller \\ Jörg Ellinger}

Department for Urology and Pediatric Urology, University Hospital Bonn,

Bonn, Germany
Correspondence: Isabella Syring Universitätsklinikum Bonn, Klinik und Poliklinik für Urologie und Kinderurologie, Sigmund-FreudStraße 25, 53। 05 Bonn, Germany Tel +49228 287 I5I09

Fax +49 228287 |4। 85

Email isabella.syring@ukb.uni-bonn.de
Abstract: Serum tumor markers have an important role in the management of patients with testicular cancer. They are useful for diagnosis, staging and risk assessment, follow-up, evaluation of response, and early detection of relapse. Alpha-fetoprotein, human chorionic gonadotropin, and lactate dehydrogenase are established serum markers in testicular cancer, but they have a limited sensitivity. Ongoing research may lead to the identification of novel biomarkers. Therefore, we review the experimental analyses for nucleic acids, circulating tumor cells, and proteins as potential biomarkers in the serum of testicular germ cell cancer patients.

Keywords: biomarker, serum, testicular germ cell cancer

\section{Introduction}

Testicular germ cell cancer (TGCT) is the most common malignant tumor in men aged 15-44 years, with its incidence rising in many countries; however, mortality rates remain low and many men are cured. ${ }^{1}$ The main factors contributing to the excellent cure rates are careful staging at the time of diagnosis, adequate early treatment based on polychemotherapy, radiotherapy, and surgery, as well as strict follow-up and salvage therapies. The introduction of the highly specific serum tumor markers alphafetoprotein (AFP) and human chorionic gonadotropin (HCG) especially improved the clinical management of patients with TGCT, and these markers nowadays play important roles in diagnosis, risk stratification, treatment monitoring, and surveillance. ${ }^{2}$ However, about $50 \%$ of TGCT patients have normally ranged tumor markers; ${ }^{3}$ thus, additional markers could be helpful for the clinician. Several researchers are working on improved noninvasive biomarkers. We will discuss recent advances in the field of circulating tumor cells (CTCs), cell-free nucleic acids, and proteins.

\section{Nucleic acids microRNA}

microRNAs (miRNAs) are small RNA molecules involved in several essential biological processes ${ }^{4}$ that cover embryogenic development, cell differentiation, apoptosis, and tumorigenesis. ${ }^{5}$ miRNAs have the potential to qualify as biomarkers in various malignancies because they mostly reveal a high stability in body fluids. ${ }^{5,6}$ Palmer et $\mathrm{al}^{7}$ recently demonstrated that miR-371-373 and miR-302 clusters are highly overexpressed in all malignant germ cell tumors (compared to normal control tissues and benign tumors), irrespective of patient age, histologic subtype, or anatomic site of the tumor. ${ }^{4}$ High expression of the miR-371-3 and miR-302 clusters 
was demonstrated in the serum of a 4-year-old boy with a yolk sac tumor and miRNA levels declined after chemotherapy. ${ }^{4}$ This finding stimulated research enormously, and Belge et al confirmed high levels of serum miR-371-3 with a standardized quantitative real-time polymerase chain reaction technique in a small cohort $(n=11)$ of TGCT patients; ${ }^{6}$ unfortunately, HCG/AFP levels were not indicated in the report. Serum levels of each of the three miRNAs (miR371, miR-372, and miR-373) decreased significantly after treatment. ${ }^{6}$ Dieckmann et al confirmed these results in a subsequent study and, most importantly, showed that the diagnostic information of the novel markers was superior to the classical tumor markers AFP and HCG (increased in four and two out of 24 TGCT patients, respectively). ${ }^{5}$ Patients in clinical stage I also showed a decline of circulating miRNAs following orchidectomy. Further research in a series with 80 TGCT patients confirmed the excellent sensitivity of serum miRNAs: ${ }^{8}$ an increase of miR-371, miR-372, miR-373, or miR-367 was observed in 98\% of the examined TGCT patients; the miRNAs thus outperformed AFP (36\%) and HCG (57\%). Our own data also confirm the improved sensitivity (85\%) and specificity (99\%) of miR-371 when compared to AFP (14\%) and HCG (37\%). ${ }^{9}$ In summary, several independent studies indicate an increase of miR-371-373 in TGCT patients irrespective of their histological subtypes and, therefore, result in a better diagnostic performance than AFP/HCG.

\section{DNA}

DNA fragments also circulate in a patient's blood, and its quantification seems to be a universal cancer biomarker. ${ }^{10}$ Earlier, we demonstrated an increase of short, presumably apoptotic DNA fragments in a cohort of 74 patients with TGCT (compared to 35 control subjects). ${ }^{11}$ The increase of genomic DNA allowed us to identify TGCT patients with a sensitivity of $84 \%$ and a specificity of $97 \% .{ }^{11}$ Whereas mitochondrial DNA is also released into circulation, the underlying mechanism seems to be different from genomic DNA: the quantification of mitochondrial DNA was less sensitive $(60 \%)$ at a similar specificity (94\%) in the same study cohort. ${ }^{12}$ Notably, the diagnostic performance of genomic and mitochondrial DNA was superior to that of AFP and HCG (24\% and 43\% sensitivity, respectively), and cell-free serum DNA levels were similar in seminoma and non-seminoma TGCT.

The analysis of circulating DNA allows for the detection of tumor-specific alterations. DNA hypermethylation is frequent during carcinogenesis, and also occurs in TGCT. Serum DNA hypermethylation in the above mentioned cohorts was detected more frequently in patients with TGCT than in healthy individuals, including $A P C=$ adenomatosis polyposis coli $57 \%$ and $6 \%, p 16(I N K) 53 \%$ and $17 \%, p 14(A R F=$ alternate reading frame) $53 \%$ and $0 \%, R A S S F 1 A=$ ras association domain family member $1 \mathrm{~A} 47 \%$ and $0 \%, P T G S 2=$ prostaglandin-endoperoxide synthase $245 \%$ and $0 \%$, and GSTP1 = glutathioneS-transferase $\pi 125 \%$ and $0 \%$, respectively. The amount of diagnostic information increased when multiple gene sites were analyzed in combination (67\% sensitivity and 97\% specificity), and was therefore superior to AFP and HCG (24\% and $43 \%$ sensitivity, respectively). ${ }^{13}$ The $\mathrm{X}$-inactive-specific transcript, which is located on the X-chromosome, is methylated in male somatic cells. The detection of unmethylated X-inactivespecific transcript DNA in plasma enabled Kawakami et al to specifically identify TGCT patients with $64 \%$ sensitivity in a cohort of 25 TCGT patients (the diagnostic accuracy of AFP/HCG was not mentioned). ${ }^{14}$ DNA methylation patterns were similar in seminoma and non-seminoma patients. ${ }^{13,14}$ So far, studies on serum/plasma DNA still require independent validation; the possible combination of DNA quantification and the assessment of qualitative alterations render cell-free DNA a study object of particular interest.

\section{CTCs}

The detection of CTCs is feasible in many malignancies, especially in patients with an advanced disease. ${ }^{15}$ Using the CellSearch assay (Veridex, Raritan, NJ, USA), CTCs were detected in the peripheral blood of $17.5 \%$ of the tested TGCT patients. Patients with advanced metastasized (clinical stage III) TGCT and non-seminoma TGCT especially showed an increase of CTCs. CTCs further correlated with AFP, HCG, and lactate dehydrogenase (LDH) levels. ${ }^{16}$ An earlier study aimed to identify CTCs via the detection of AFP and HCG messenger RNA (mRNA) using real-time polymerase chain reaction in peripheral blood cells: overall, $27 \%$ of patients had an increase of these mRNA, and there was also a correlation of AFP/HCG mRNA positivity with clinical stage IIC/III. ${ }^{17}$ Given the low frequency of CTCs in patients with minimal tumor burden, there seems to be only a limited potential for CTCs in the clinical management of patients with TGCT. Furthermore, serum AFP (25\%) and HCG (49\%) provided a similar or even better sensitivity.

\section{Proteins \\ TRA- I-60}

A report in 1991 suggested that terato-related antigen monoclonal antibody (TRA-1-60) could serve as a tumor marker for 
TGCT patients with an embryonal carcinoma component. ${ }^{18}$ The analysis of TRA-1-60 in stage I non-seminoma TGCT indicated similar diagnostic performance as the conventional markers. However, during follow-up, TRA-1-60 levels were increased 1 month before radiological signs of recurrence and decreased during treatment. ${ }^{19}$ A subsequent study by Lajer et al confirmed an increase of TRA-1-60 in TGCT patients, but the clinical utility was limited by a high rate of false-positive elevations. ${ }^{20}$

\section{Neuron-specific enolase (NSE)}

NSE, an isoenzyme of the glycolytic enzyme 2-phospho-Dglycerate-hydrolase, is a marker for tumors of neuroendocrine origin. It is recognized as a useful tumor marker in smallcell lung cancer and melanoma. ${ }^{21}$ Early studies indicated an increase of NSE in seminoma TGCT, and the diagnostic sensitivity and specificity were in the same order as those of HCG. ${ }^{22,23}$ However, large-scale studies have shown frequent false-positive or false-negative results of NSE during follow-up examinations, and thus do not support NSE to be a valuable tumor marker in germ-cell tumors. ${ }^{24,25}$ Furthermore, hemolysis leads to an elevation of serum NSE. ${ }^{25}$ Accordingly, Sturgeon et al point out that in spite of promising results (30\%-50\% positivity of patients with seminomas and less often in non-seminomatous germ cell tumor patients), the use of NSE is limited and, so far, only in an experimental stage of development. ${ }^{26}$

\section{Lectin (LCA)-reactive AFP}

It is sometimes difficult to decide on a treatment plan for seminoma patients with slightly increased AFP levels as these could also be due to liver disease. LCA-reactive AFP, determined by LCA-affinity electrophoresis, might be a specific tool to identify AFP derived from undetected embryonal cell carcinoma or yolk sac tumor elements. ${ }^{27}$ Furthermore, Kawai et al showed that LCA-reactive AFP was detected after the normalization of total AFP levels in stage I TGCT patients $(n=3)$ who suffered from tumor recurrence. ${ }^{27}$

\section{Fas and Fas ligand}

Fas/Apo-1, a member of the tumor necrosis factor receptor superfamily, can transduce a signal that leads to the rapid induction of apoptosis by binding the Fas ligand (FasL). Serum levels of soluble FasL are increased in patients with various malignancies. ${ }^{28}$ In TGCT patients with a seminoma component, Fas and FasL are expressed in 73\% of tumor tissues, whereas its expression is only $56 \%$ for Fas and $11 \%$ for FasL in non-seminoma tissue. ${ }^{29}$ An elevation of soluble
FasL was noticed in $38 \%$ of patients with seminomatous TGCT elements $(n=24) .{ }^{29}$ Unfortunately, the sensitivity of HCG was not provided in the study cohort.

\section{CD30}

CD30 (TNFRSF8) is a protein of the tumor necrosis factor receptor family. It can be detected in extracellular fluids (eg, anaplastic large-cell lymphoma) ${ }^{30}$ and it correlates with the tumor burden. CD30 is expressed exclusively in embryonal carcinoma components of TGCT. ${ }^{31}$ Latza et al reported soluble CD30 in the serum of all patients $(n=8)$ with embryonal cell carcinoma, but only in $20 \%$ of patients with other TGCT components. ${ }^{30}$ Moreover, serum levels of soluble CD30 antigen seem to be a promising parameter for monitoring patients with embryonal cell carcinoma. ${ }^{30}$ Persistent CD30 expression in embryonal carcinoma cells after cisplatin-based chemotherapy is indicative of recurrent and progressive $\mathrm{TGCT}^{32}$ and monitoring this protein in the serum may be helpful for clinical decision making.

\section{C-reactive protein (CRP)}

CRP is an acute-phase reactant and a useful marker of systemic inflammation. ${ }^{33}$ Systemic inflammation has been linked to cancer progression, and, in patients with urological cancers, the presence of a systemic inflammatory response is thought to be indicative of a poor prognosis. However, CRP has not been studied as a prognostic biomarker in TGCT. Furthermore, CRP levels have been proven to increase after radiotherapy in contrast to chemotherapy or surgery alone, and it is speculated that a CRP increase could be predictive of late complications like cardiovascular disease ${ }^{34}$ and chronic cancer-related fatigue. ${ }^{33}$

\section{Free $\beta$-subunit of $\mathrm{HCG}(\mathrm{HCG} \beta)$}

In the serum of patients with seminoma, HCG is increased in only about $15 \%-20 \%$ of the cases. However, $30 \%-40 \%$ of seminoma patients have been found to have increased serum concentrations of HCG $\beta .^{35}$ The determination of HCG $\beta$ increases the frequency of marker-positive seminoma from $17 \%$ to $57 \%$ and of marker-positive relapses from $32 \%$ to $59 \%$. But still, about $40 \%$ of marker-positive seminoma and relapses would have been missed with an assay measuring $\mathrm{HCG}$ and $\mathrm{HCG}$ together. In patients with seminomatous testicular cancer, HCGß is superior to HCG, and in some non-seminomatous germ cell tumor patients it provides additional information. ${ }^{35}$ Notably, an increase of both HCGß and HCG was in most cases limited to patients with a choriocarcinomatous component, whereas 
the majority of seminoma patients had only an increase of HCGß but not HCG. ${ }^{35}$ The lack of HCGß may therefore be indicative of choriocarcinoma.

\section{Hyperglycosylated HCG (HCG-h)}

HCG-h contains larger and more complex carbohydrate chains than regular HCG. ${ }^{36}$ Lempiäinen et al showed that a major proportion of $\mathrm{HCG}$ was hyperglycosylated preoperatively, at relapse, and shortly after treatment. ${ }^{36}$ The serum concentrations of HCG-h and HCG correlated strongly with each other and had similar diagnostic value. HCG-h does not appear to provide clinical information additional to that obtained by conventional HCG assays. ${ }^{36}$

\section{Angiogenic growth factors (pleiotrophin [PTN] and fibroblast growth factor-2 [FGF-2])}

Increased serum concentrations of various angiogenic growth factors have been described as potential markers for tumor progression and metastasis in other tumor entities. ${ }^{37}$ Various angiogenic growth factors (ie, FGF-2, vascular endothelial growth factor, FGF-4, transforming growth factor-beta, epidermal growth factor, and PTN) have been analyzed by Aigner et al in TGCT. ${ }^{37}$ Significantly elevated levels of FGF-2 and PTN were observed in the serum of testicular cancer patients, and FGF-2 serum levels especially allowed TGCT patients $(n=22)$ and normal individuals $(n=21)$ to be distinguished very accurately (sensitivity/specificity was not reported). FGF-2 serum levels were also elevated in early tumor stages. In comparison, AFP was elevated in $66 \%$ of non-seminoma patients, and HCG was increased in $23 \%$ of seminoma patients and $56 \%$ of non-seminoma patients. The data indicate that serum levels of FGF-2 and PTN might be useful novel tumor markers but confirmative studies are required.

\section{Circulating full-length and caspase- cleaved cytokeratin 18 (CKI8)}

Circulating full-length and caspase-cleaved CK18 are considered biomarkers of chemotherapy-induced cell death measured using a combination of the M30 and M65 enzyme-linked immunosorbent assays. M30 measures caspase-cleaved CK18 produced during apoptosis and M65 measures the levels of both caspase-cleaved and intact CK18, the latter of which is released from cells undergoing necrosis. ${ }^{38}$ During necrotic and apoptotic cell death, CK18 and other cytokeratins are released into the blood in either their intact or their caspase-cleaved forms. There they remain relatively stable in the circulation of patients with cancer. Previous studies have highlighted their potential as prognostic, predictive, and pharmacological tools in the treatment of cancer. M65 and M30 levels appear to reflect chemotherapy-induced changes that correlate with changes in markers routinely used in the clinic for managing patients with non-seminomatous TGCT. ${ }^{38}$ The overall decreases in M65 and M30 are indicative of treatment response, as they appear to reflect a decrease in tumor load due to chemotherapy-induced tumor cell death. The correlation between M65/M30 levels and International Germ Cell Consensus Classification (IGCCC) prognosis groups, as well as their overall agreement with $\mathrm{LDH}$, AFP, and HCG levels, suggest that M65/M30 may also have prognostic value in patients with non-seminomatous TCGT. ${ }^{38}$ Although some patients were recruited prospectively in this study, additional studies are needed to underline the benefit of M65/M30 measurements during chemotherapeutic treatment.

\section{Conclusion}

The established serum tumor markers AFP, HCG, and LDH play an important role in the clinical management of TGCT patients; but because of their limited sensitivity, additional markers are needed. Very promising novel markers such as circulating microRNAs or FGF-2, which are detectable at similar frequencies in seminoma/non-seminoma TGCT patients and are absent in healthy subjects, are of particular interest as diagnostic tools. The analysis of M65/M30 may provide additional information as a treatment monitoring marker. Nevertheless, validating studies in large-scale, prospective, and clinically relevant patient cohorts are urgently needed. The detection technique of serum miR-371 is being optimized by a startup at the University of Bremen (miRdetect), and prospective studies to prove the clinical use of this marker are to be expected within the next few years.

\section{Disclosure}

The authors report no conflicts of interest in this work.

\section{References}

1. Shanmugalingam T, Soultati A, Chowdhury S, Rudman S, Van Hemelrijck M. Global incidence and outcome of testicular cancer. Clin Epidemiol. 2013;5:417-427.

2. Salem M, Gilligan T. Serum tumor markers and their utilization in the management of germ-cell tumors in adult males. Expert Rev Anticancer Ther. 2011;11(1):1-4.

3. Albers P, Albrecht W, Algaba F, et al; European Association of Urology. EAU guidelines on testicular cancer: 2011 update. Eur Urol. 2011;60(2):304-319.

4. Murray MJ, Halsall DJ, Hook CE, Williams DM, Nicholson JC, Coleman N. Identification of microRNAs From the miR-371 373 and miR-302 clusters as potential serum biomarkers of malignant germ cell tumors. Am J Clin Pathol. 2011;135(1):119-125.

5. Dieckmann KP, Spiekermann M, Balks T, et al. MicroRNAs miR-371-3 in serum as diagnostic tools in the management of testicular germ cell tumours. Br J Cancer. 2012;107(10):1754-1760. 
6. Belge G, Dieckmann KP, Spiekermann M, Balks T, Bullerdiek J. Serum levels of microRNAs miR-371-3: a novel class of serum biomarkers for testicular germ cell tumors? Eur Urol. 2012;61(5):1068-1069.

7. Palmer RD, Murray MJ, Saini HK, et al; Children's Cancer and Leukaemia Group. Malignant germ cell tumors display common microRNA profiles resulting in global changes in expression of messenger RNA targets. Cancer Res. 2010;70(7):2911-2923.

8. Gillis AJ, Rijlaarsdam MA, Eini R, et al. Targeted serum miRNA (TSmiR) test for diagnosis and follow-up of (testicular) germ cell cancer patients: a proof of principle. Mol Oncol. 2013;7(6):1083-1092.

9. Syring I, Bartels J, Holdenrieder S, Kristiansen G, Müller SC, Ellinger J. Circulating serum micro RNA (miR-367-3p, miR-371a-3p, miR372-3p, miR-373-3p) as biomarkers for patients with testicular germ cell cancers. J Urol. Epub July 2014 (in press).

10. Ellinger J, Müller SC, Stadler TC, Jung A, von Ruecker A, Bastian PJ. The role of cell-free circulating DNA in the diagnosis and prognosis of prostate cancer. Urol Oncol. 2011;29(2):124-129.

11. Ellinger J, Wittkamp V, Albers P, et al. Cell-free circulating DNA: diagnostic value in patients with testicular germ cell cancer. $J$ Urol. 2009;181(1):363-371.

12. Ellinger J, Albers P, Müller SC, von Ruecker A, Bastian PJ. Circulating mitochondrial DNA in the serum of patients with testicular germ cell cancer as a novel noninvasive diagnostic biomarker. BJU Int. 2009;104(1):48-52.

13. Ellinger J, Albers P, Perabo FG, Müller SC, von Ruecker A, Bastian PJ. $\mathrm{CpG}$ island hypermethylation of cell-free circulating serum DNA in patients with testicular cancer. J Urol. 2009;182(1):324-329.

14. Kawakami T, Okamoto K, Ogawa O, Okada Y. XIST unmethylated DNA fragments in male-derived plasma as a tumour marker for testicular cancer. Lancet. 2004;363(9402):40-42.

15. Yuasa T, Yoshiki T, Tanaka T, Isono T, Okada Y. Detection of circulating testicular cancer cells in peripheral blood. Cancer Lett. 1999;143(1): 57-62.

16. Nastaly P, RufCG, Becker P, et al. Circulating tumor cells in patients with testicular germ cell tumors. Clin Cancer Res. Epub March 14, 2014.

17. Hautkappe AL, Lu M, Mueller H, et al. Detection of germ-cell tumor cells in the peripheral blood by nested reverse transcription-polymerase chain reaction for alpha-fetoprotein-messenger RNA and beta human chorionic gonadotropin-messenger RNA. Cancer Res. 2000;60(12) 3170-3174.

18. Marrink J, Andrews PW, van Brummen PJ, et al. TRA-1-60: a new serum marker in patients with germ-cell tumors. Int $J$ Cancer. 1991;49(3):368-372.

19. Gels ME, Marrink J, Visser P, et al. Importance of a new tumor marker TRA-1-60 in the follow-up of patients with clinical stage I nonseminomatous testicular germ cell tumors. Ann Surg Oncol. 1997;4(4): 321-327.

20. Lajer H, Daugaard G, Andersson AM, Skakkebaek NE. Clinical use of serum TRA-1-60 as tumor marker in patients with germ cell cancer. Int $J$ Cancer. 2002;100(2):244-246.

21. Cooper EH. Neuron-specific enolase. Int J Biol Markers. 1994;9(4): 205-210.

22. Fosså SD, Klepp O, Paus E. Neuron-specific enolase - a serum tumour marker in seminoma? Br J Cancer. 1992;65(2):297-299.
23. Kuzmits R, Schernthaner G, Krisch K. Serum neuron-specific enolase A marker for responses to therapy in seminoma. Cancer. 1987;60(5): 1017-1021.

24. Gross AJ, Dieckmann KP. Neuron-specific enolase: a serum tumor marker in malignant germ-cell tumors? Eur Urol. 1993;24(2):277-278.

25. Tandstad T, Klepp O. Neuron-specific enolase in testicular cancer clinical experiences with serum neuron-specific enolase in patients with testicular cancer at diagnosis and during follow-up. Acta Oncol. 2003;42(3):202-206.

26. Sturgeon CM, Duffy MJ, Stenman UH, et al; National Academy of Clinical Biochemistry. National Academy of Clinical Biochemistry laboratory medicine practice guidelines for use of tumor markers in testicular, prostate, colorectal, breast, and ovarian cancers. Clin Chem. 2008;54(12):e11-e79.

27. Kawai K, Kojima T, Miyanaga N, et al. Lectin-reactive alpha-fetoprotein as a marker for testicular tumor activity. Int J Urol. 2005;12(3): 284-289.

28. Nakamoto Y, Kaneko S, Buttner SW, Matsushita E, Kobayashi K. Inhibition of peripheral blood lymphocyte apoptosis by soluble fas ligand in patients with hepatocellular carcinoma. Oncol Res. 1999;6(4):733-739.

29. Hara S, Miyake H, Nakamura I, Arakawa S, Kamidono S, Hara I. Increased Fas ligand expression in the tumor tissue and serum of patients with testicular germ cell tumors with seminomatous elements. Urology. 2001;58(3):471-476.

30. Latza U, Foss HD, Dürkop H, et al. CD30 antigen in embryonal carcinoma and embryogenesis and release of the soluble molecule. Am J Pathol. 1995;146(2):463-471.

31. Teng LH, Lu DH, Xu QZ, Fu YJ, Yang H, He ZL. [Expression and diagnostic significance of OCT4, CD117 and CD30 in germ cell tumors]. Zhonghua Bing Li Xue Za Zhi. 2005;34(11):711-715. Chinese.

32. Giannatempo P, Paolini B, Miceli R, et al. Persistent CD30 expression by embryonal carcinoma in the treatment time course: prognostic significance of a worthwhile target for personalized treatment. J Urol. 2013;190(5):1919-1924.

33. Saito K, Kihara K. C-reactive protein as a biomarker for urological cancers. Nat Rev Urol. 2011;8(12):659-666.

34. Danesh J, Wheeler JG, Hirschfield GM, et al. C-reactive protein and other circulating markers of inflammation in the prediction of coronary heart disease. N Engl J Med. 2004;350(14):1387-1397.

35. Lempiäinen A, Stenman UH, Blomqvist C, Hotakainen K. Free betasubunit of human chorionic gonadotropin in serum is a diagnostically sensitive marker of seminomatous testicular cancer. Clin Chem. 2008;54(11):1840-1843.

36. Lempiäinen A, Hotakainen K, Blomqvist C, Alfthan H, Stenman UH. Hyperglycosylated human chorionic gonadotropin in serum of testicular cancer patients. Clin Chem. 2012;58(7):1123-1129.

37. Aigner A, Brachmann P, Beyer J, et al. Marked increase of the growth factors pleiotrophin and fibroblast growth factor- 2 in serum of testicular cancer patients. Ann Oncol. 2003;14(10):1525-1529.

38. de Haas EC, di Pietro A, Simpson KL, et al. Clinical evaluation of M30 and M65 ELISA cell death assays as circulating biomarkers in a drug-sensitive tumor, testicular cancer. Neoplasia. 2008;10(10): 1041-1048.

Current Biomarker Findings

\section{Publish your work in this journal}

Current Biomarker Findings is an international, peer-reviewed, open access journal publishing original research, reports, reviews and commentaries on all areas of biomarker research. The manuscript management system is completely online and includes a very quick and fair

peer-review system. Visit http://www.dovepress.com/testimonials.php to read real quotes from published authors. 